\title{
IgG4-related disease in pulmonary arterial hypertension on long-term epoprostenol treatment
}

\author{
To the Editor:
}

IgG4-related disease (IgG4-RD) is a recently described systemic fibro-inflammatory condition characterised by tumefactive lesions and dense lymphoplasmacytic infiltrates rich in IgG4-positive plasma cells [1]. IgG4$\mathrm{RD}$ has been described in a variety of organ systems, including the lacrimal gland, salivary gland, lungs, pancreas and kidneys, and often responds favourably to corticosteroids. The pathophysiological mechanisms for IgG4-RD remain unclear, but type 2 T-helper (Th2) cells are thought to play a critical role $[2,3]$. We recently encountered a patient who developed IgG4-RD during the course of idiopathic pulmonary arterial hypertension $(\mathrm{PAH})$. After this experience, we carefully examined PAH patients for IgG4-RD and found four additional cases.

The index case patient was a 19-year-old female who presented with progressive exertional dyspnoea corresponding to World Health Organization functional class (WHO-FC) II in March 2000. She was referred to Keio University Hospital (Tokyo, Japan), where complete pulmonary hypertension ( $\mathrm{PH}$ ) evaluations were conducted. Right heart catheterisation revealed an elevated mean pulmonary arterial pressure (mPAP) of $67 \mathrm{mmHg}$ and a normal pulmonary arterial wedge pressure of $7 \mathrm{mmHg}$. There was no condition or underlying disease that accounted for her PH, leading to the diagnosis of idiopathic PAH. She was initially treated with warfarin and nifedipine, and an oral prostacyclin analogue, beraprost, was added in January 2002. 1 year later, her dyspnoea had deteriorated (to WHO-FC III), with marked elevation of the mPAP (to $95 \mathrm{mmHg}$ ). Beraprost was replaced by epoprostenol, and the dosage was gradually increased to $57 \mathrm{ng} \cdot \mathrm{kg}^{-1} \cdot \mathrm{min}^{-1}$, resulting in improved symptoms and haemodynamic parameters (mPAP $47 \mathrm{mmHg}$ ).

In January 2007, the patient developed chronic sinusitis. Simultaneously, she noticed a gradual enlargement of bilateral lacrimal, parotid and submandibular glands, and of minor salivary glands of the lips, in conjunction with intermittent upper abdominal pain (fig. la-g). A computed tomography scan revealed diffuse enlargement of bilateral lacrimal and salivary glands and pancreas. Her serum IgG4 level was remarkably elevated (to $824 \mathrm{mg} \cdot \mathrm{dL}^{-1}$ from a normal range of $<135 \mathrm{mg} \cdot \mathrm{dL}^{-1}$ ). Histopathological analysis of specimens obtained from labial glands showed a dense lymphoplasmacytic infiltrate around the ducts and acini. The inflammatory infiltrate was mainly composed of $\mathrm{CD} 20^{+} \mathrm{B}$-cells and $\mathrm{CD} 138^{+}$plasma cells. The infiltrating plasma cells were predominantly IgG4-bearing cells, which exceeded $50 \%$ of the total plasma cells. The diagnosis was IgG4-related dacryoadenitis, sialadenitis and pancreatitis. The patient was treated with $35 \mathrm{mg}$ prednisolone daily and showed prompt improvement of the tumefactive lesions. However, 4 months later, she died of uncontrolled right heart failure due to exacerbation of PAH.

After we encountered the index case in 2007, we examined PAH patients who were being followed at the specialised PH clinic of Keio University Hospital for IgG4-RD. Between 2008 and 2012, we found four additional cases of idiopathic/heritable PAH with complicating IgG4-RD during the disease course. To examine the incidence rate, clinical characteristics and predisposing factors for IgG4-RD in patients with $\mathrm{PAH}$, we retrospectively obtained clinical information for 75 consecutive patients with idiopathic/heritable $\mathrm{PAH}$ and 40 consecutive patients with PAH associated with connective tissue disease (CTD), who first visited the specialised PH clinic of Keio University Hospital between 2000 and 2009. IgG4-RD was diagnosed based on the characteristic organ involvement with elevated serum IgG4 level and/or typical histopathological findings [4]. All the clinical information was collected in June 2012. The study was approved by the Keio University Institutional Review Board.

IgG4-RD was observed in five (6.7\%) out of the 75 patients with idiopathic/heritable $\mathrm{PAH}$, but in none of the 40 patients with PAH-CTD. None of the patients without IgG4-RD showed enlarged lacrimal or salivary glands upon careful physical examination, or an increase in serum IgG4. The incidence rate of IgG4-RD in patients with idiopathic/heritable PAH was 14.3 per 1000 patient-years. Since the estimated prevalence of IgG4-RD in Japan was $0.28-1.08$ per 100000 in a community-based study [5], patients with idiopathic/ heritable PAH are at extremely high risk for developing IgG4-RD. 

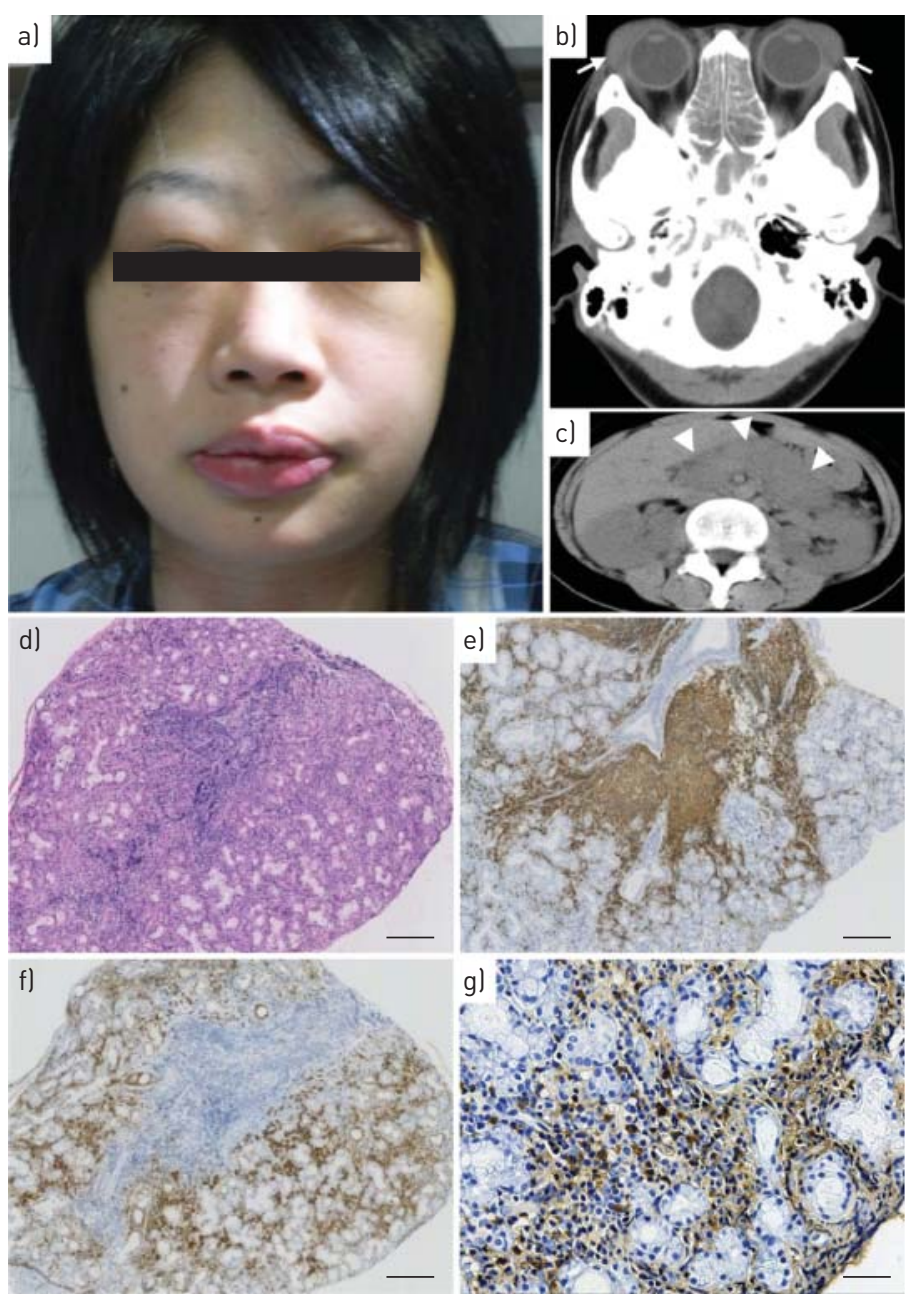
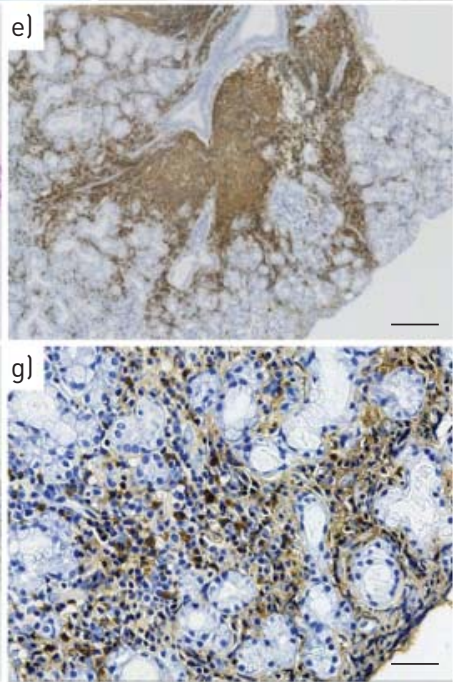
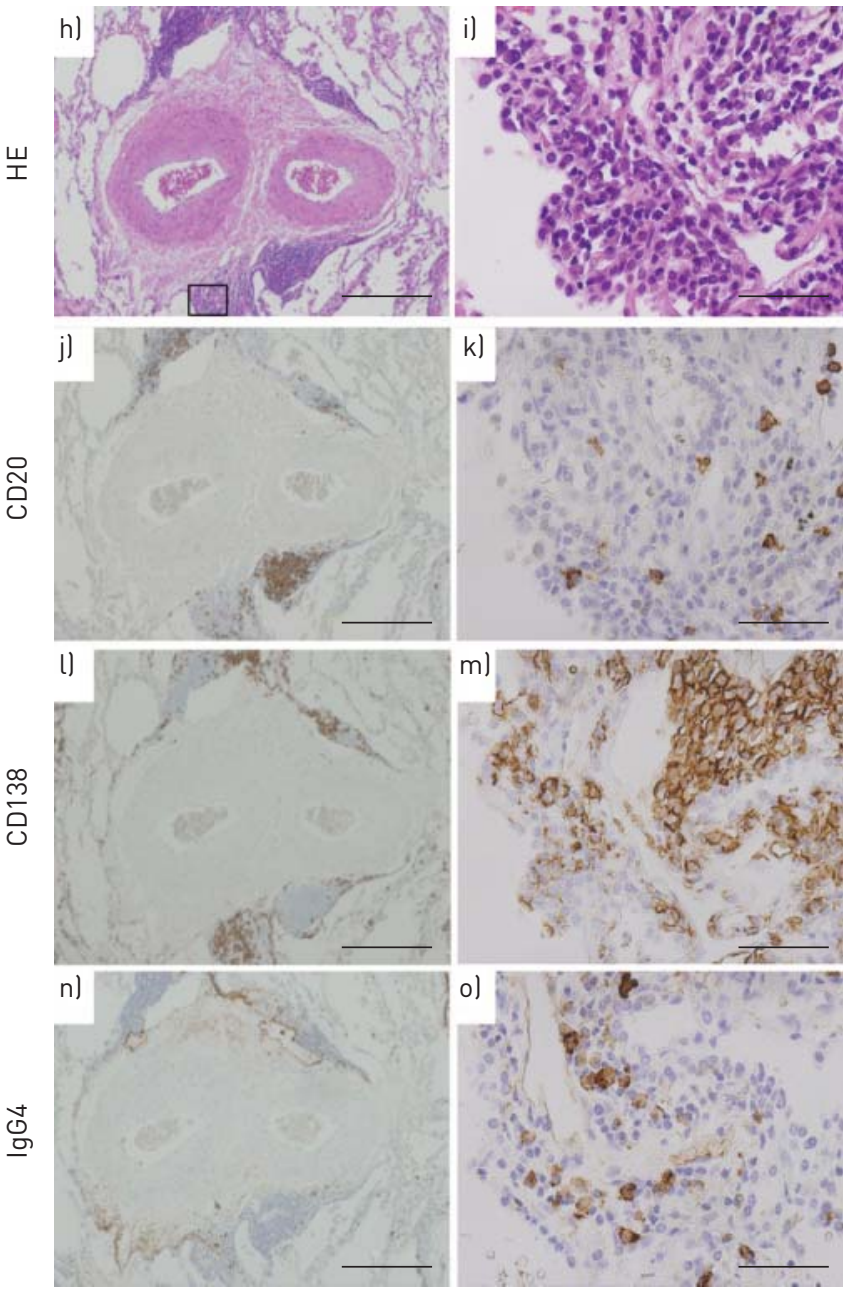

FIGURE $1 \mathrm{a}-\mathrm{g}$ ) Clinical, radiological and histopathological findings of the index case (case 1) and h-o) histopathological findings of the consecutive lung sections from case 3. a) Bilateral enlargement of the lacrimal, parotid and submandibular glands. Lips of the mouth were markedly swollen because of beaded enlargement of the labial glands. b) Computed tomography (CT) scan showed diffusely enlarged bilateral lacrimal glands (arrows). c) CT scan showed a diffusely enlarged pancreas (arrowheads). d) Haematoxylin and eosin (HE) staining of a labial gland specimen obtained from lip biopsy. A dense lymphoplasmacytic infiltrate was found in the interstitium around the ducts and acini. e) CD20 immunostaining of the labial gland specimen showed B-cell accumulation predominantly around the duct. f) CD138 immunostaining of the labial gland specimen showed diffuse infiltrates of plasma cells in the peri-acinal area. $g$ ) IgG4 immunostaining of the labial gland specimen showed a predominance of IgG4-bearing plasma cells. h, i) HE staining showed a focal and dense lymphoplasmacytic infiltrate around the muscular pulmonary arteries with medial and adventitial thickening. $\mathrm{h}$ ) Box indicates part of image that is shown at higher magnification in i, $\mathrm{k}$, $\mathrm{m}$ and o. j, $\mathrm{k}$ ) CD20 immunostaining showed focal B-cell accumulation predominantly around the narrowed pulmonary arteries. 1 , m) CD138 immunostaining showed focal

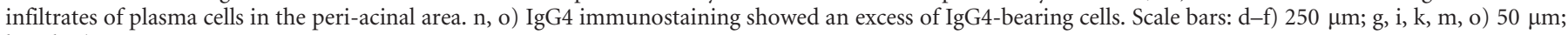
h, j, l, n) $500 \mu \mathrm{m}$.

Table 1 summarises clinical characteristics of the five patients with idiopathic/heritable PAH who developed IgG4-RD. Interestingly, IgG4-RD occurred during the course of PAH, between 65 and 125 months after diagnosis of PAH. The lacrimal and salivary glands were symmetrically affected in all five cases, consistent with a previously recognised condition called Mikulicz disease, while none of them had sicca syndrome or positive anti-SSA or anti-SSB antibodies, which are typical features of Sjögren's syndrome. Pancreas involvement was also detected in two patients. Concomitant onset of allergic diseases was common.

As treatment for the IgG4-RD, four patients received corticosteroids (prednisolone $>0.5 \mathrm{mg} \cdot \mathrm{kg}^{-1}$ daily), and the remaining patient (case 3) was not treated because of an upcoming lung transplant. Histopathological analysis of lung specimens obtained at transplantation showed focal and dense lymphoplasmacytic infiltrates around the muscular pulmonary arteries with medial and adventitial thickening. The infiltrate consisted mainly of $\mathrm{CD} 20^{+} \mathrm{B}$-cells and $\mathrm{CD} 138^{+}$plasma cells with an excess of IgG4-bearing cells (fig. 1h-O). Favourable responses, including resolution of tumorous swellings and decrease in serum IgG4, were observed in all patients who received corticosteroids, but none of them 
TABLE 1 Clinical characteristics of patients with idiopathic/heritable pulmonary arterial hypertension (PAH) who developed IgG4-related disease (IgG4-RD) during the course of the disease

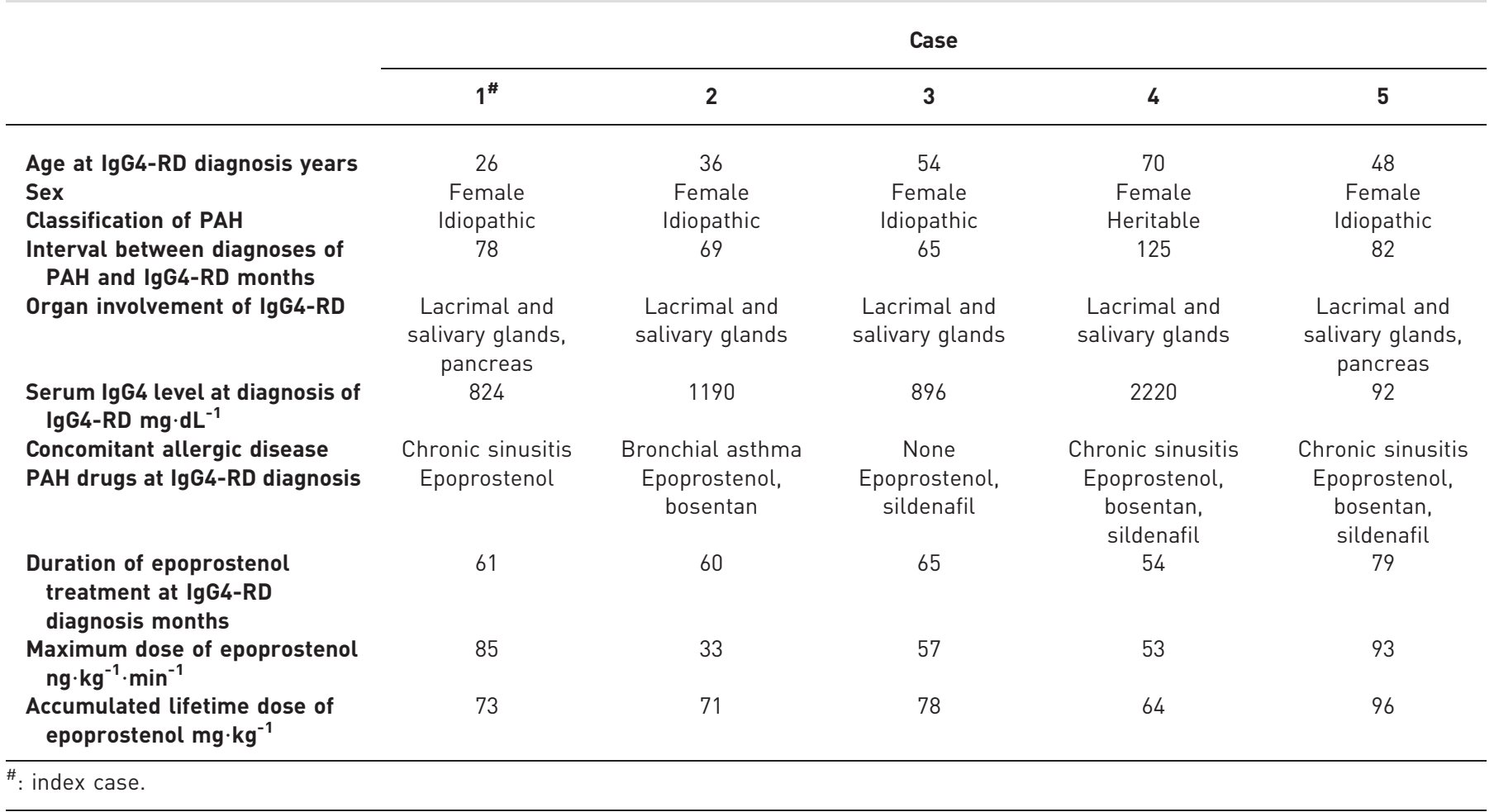

showed improvement in PAH. None experienced recurrence of IgG4-RD, and one patient died of exacerbation of the PAH.

Comparisons of the demographic and clinical characteristics of 75 patients with idiopathic/heritable $\mathrm{PAH}$ stratified by the presence or absence of IgG4-RD revealed no significant difference in age, sex, WHO-FC or haemodynamic parameters at the time of PAH or IgG4-RD diagnosis. Regarding the drugs used to treat the $\mathrm{PAH}$, all five patients were on epoprostenol when IgG4-RD was diagnosed, while 46 out of 70 patients without IgG4-RD were treated with epoprostenol ( $\mathrm{p}=0.16$, Fisher's exact test). Notably, long-term treatment with a high concentration of epoprostenol appeared to be a risk factor for development of IgG4RD. Specifically, the relative risk for developing IgG4-RD was $>14.4$ in patients who had received epoprostenol treatment for $>4$ years $(p=0.002)$, and was $>10.2$ in patients with accumulated lifetime dose of epoprostenol $>50 \mathrm{mg} \cdot \mathrm{kg}^{-1}(\mathrm{p}=0.006)$.

This is the first report describing a potential link between PAH and IgG4-RD. Since PAH is observed in association with various inflammatory conditions, such as CTD, one could speculate that PAH is one of the manifestations of IgG4-RD. In this regard, lung specimens obtained from one of the cases showed histological features compatible with IgG4-RD, but it remains unclear how lymphoplasmacytic infiltration present outside the vasculature contributes to remodelling of pulmonary arteries. IgG4-RD occurred $>5$ years after diagnosis of $\mathrm{PAH}$, when the WHO-FC and haemodynamics were stable. In addition, corticosteroid treatment dramatically improved IgG4-RD but was ineffective for PAH, although rituximab, a potential agent effective for refractory IgG4-RD [6], was not tried for any of our patients. Conversely, long-term treatment with a high epoprostenol dose was suggested as a risk for IgG4-RD. Epoprostenol, a synthetic prostacyclin analogue, exerts its function mainly through a prostaglandin $\mathrm{I}_{2}$ (IP) receptor, but also through other prostaglandin receptors such as DP1 and EP2 [7]. These receptors are reported to modulate development and function of T-helper cells [8], such as enhancement of Th2 expansion [9, 10]. It is intriguing to speculate that long-term exposure to a high concentration of epoprostenol contributes to IgG4-RD development by shifting the T-helper cell balance to Th2. However, further studies accumulating similar cases are necessary to clarify a causal relationship between these two conditions.

@ERSpublications

IgG4-related disease may be associated with patients with $\mathrm{PAH}$, especially those treated with longterm epoprostenol http://ow.ly/rFwlO 
Yuichiro Shirai ${ }^{1}$, Yuichi Tamura ${ }^{2}$, Hidekata Yasuoka ${ }^{1}$, Toru Satoh ${ }^{2,3}$ and Masataka Kuwana ${ }^{1}$

${ }^{1}$ Division of Rheumatology, Dept of Internal Medicine, Keio University School of Medicine, Tokyo, ${ }^{2}$ Dept of Cardiology, Keio University School of Medicine, Tokyo, and ${ }^{3}$ Division of Cardiology, Kyorin University School of Medicine, Tokyo, Japan.

Correspondence: M. Kuwana, Division of Rheumatology, Keio University School of Medicine, 35 Shinanomachi, Shinjuku-ku, Tokyo 160-8582, Japan. E-mail: kuwanam@z5.keio.jp

Received: Aug 162013 | Accepted after revision: Dec 022013 | First published online: Jan 162014

Support statement: This work was supported by a research grant (no. 24131701) on intractable diseases from the Japanese Ministry of Health, Labour and Welfare. The funder had no role in the analysis of data and decision to publish.

Conflict of interest: Disclosures can be found alongside the online version of this article at www.erj.ersjournals.com

\title{
References
}

Stone JH, Zen Y, Deshpande V. IgG4-related disease. N Eng J Med 2012; 366: 539-551.

2 Kanari H, Kagami S, Kashiwakuma D, et al. Role of Th2 cells in IgG4-related lacrimal gland enlargement. Int Arch Allergy Immunol 2010; 152: Suppl. 1, 47-53.

3 Tanaka A, Moriyama $\mathrm{M}$, Nakashima $\mathrm{H}$, et al. Th2 and regulatory immune reactions contribute to IgG4 production and the initiation of Mikulicz disease. Arthritis Rheum 2012; 64: 254-263.

4 Umehara H, Okazaki K, Masaki Y, et al. Comprehensive diagnostic criteria for IgG4-related disease (IgG4-RD), 2011. Mod Rheumatol 2012; 22: 21-30.

5 Umehara H, Okazaki K, Masaki Y, et al. A novel clinical entity, IgG4-related disease (IgG4RD): general concept and details. Mod Rheumatol 2012; 22: 1-14.

$6 \quad$ Khosroshahi A, Bloch DB, Deshpande V, et al. Rituximab therapy leads to rapid decline of serum IgG4 levels and prompt clinical improvement in IgG4-related systemic disease. Arthritis Rheum 2010; 62: 1755-1762.

7 Whittle BJ, Silverstein AM, Mottola DM, et al. Binding and activity of the prostacyclin receptor (IP) agonists, treprostinil and iloprost, at human prostanoid receptors: treprostinil is a potent DP1 and EP2 agonist. Biochem Pharmacol 2012; 84: 68-75.

8 Hata AN, Breyer RM. Pharmacology and signaling of prostaglandin receptors: multiple roles in inflammation and immune modulation. Pharmacol Ther 2004; 103: 147-166.

9 Boswell MG, Zhou W, Newcomb DC, et al. PGI2 as a regulator of CD4+ subset differentiation and function. Prostaglandins Other Lipid Mediat 2011; 96: 21-26.

10 Kuo CH, Ko YC, Yang SN, et al. Effects of PGI2 analogues on Th1- and Th2-related chemokines in monocytes vio epigenetic regulation. J Mol Med 2011; 89: 29-41.

\section{Point-of-care urine test for assessing adherence to isoniazid treatment for tuberculosis}

\author{
To the Editor:
}

Good adherence to treatment for tuberculosis (TB) is essential, both to cure disease and prevent development of drug resistance. Adherence to chemoprophylaxis (preventive therapy) for latent $\mathrm{TB}$ infection (LTBI) is particularly poor [1].

Methods for measuring adherence to TB medication include detecting urine colour change due to the presence of rifampicin. However, this effect is short lived, peaking at $2-6 \mathrm{~h}$ and is only seen in $<50 \%$ of patients [2]. Directly observed therapy (DOT) will ensure adherence to antituberculous treatment, but this can be unacceptable and many patients do not tolerate a three times a week regimen. DOT is costly in terms of personnel and is seldom employed in chemoprophylaxis patients [1]. The highly reliable Arkansas method for detecting isoniazid metabolites in urine relies on a laboratory colorimetric assay, involving adding drops of prepared solutions of reagents, including potassium cyanide, to a urine sample [3]. There are obvious risks involved in handling and storing the reagents.

IsoScreen (GFC Diagnostics, Bicester, UK) uses the reagents of the Arkansas Method but in an enclosed plastic testing device (SafeTube; GFC Diagnostics), allowing safe and rapid point-of-care testing in clinics and patients' homes [2]. The urine colour change is apparent within a few seconds but 5 min is allowed to ensure stable colour development. Purple or blue samples are deemed positive, green intermediate and 